\title{
GLOBAL THEOREMS FOR CLOSED PLANE CURVES
}

BY BENJAMIN HALPERN ${ }^{1}$

Communicated by O. Zariski, May 21, 1969

Let $X$ be a closed plane curve given by a four times continuously differentiable map $X: C \rightarrow R^{2}$ from the circle $C$ into Euclidean twodimensional space $R^{2}$. The results announced here are typified by

THEOREM 1. Under certain regularity conditions, the number of straight lines which are tangent to $X$ at two points $s$ and $t$ of $X$ and such that the unit normals to $X$ at $s$ and $t$ are equal is equal to the number of straight lines which are tangent to $X$ at two points s and $t$ of $X$ and such that the unit normals to $X$ at $s$ and t are unequal, plus the number of selfintersections of $X$, plus one-half the number of inflection points of $X$. In Figure 1 the double tangents of the first mentioned kind are drawn solid and the others are dashed.

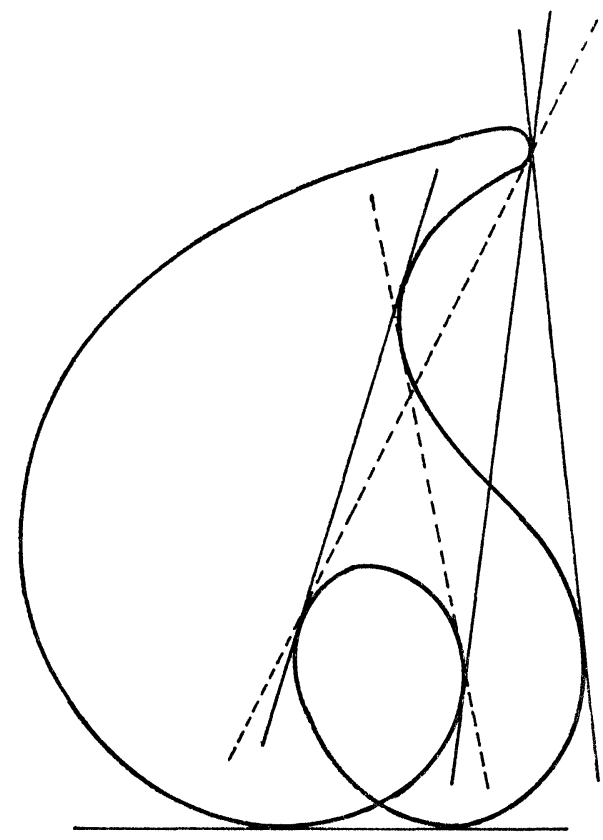

FIGURE 1

1 This research was supported in part by the National Science Foundation, Grant No. GP8397. 
The formulas presented here may be thought of as analogs to the classical Plücker formulas for complex algebraic curves.

Definitions AND NOtations. It is assumed that the tangent to $X, X^{\prime}(s)$ never vanishes. For each $s \in C$ let $\kappa(s), \rho(s), c(s), n(s), N_{s}$ and $T_{s}$ be the curvature, radius of curvature, center of curvature, unit normal, normal line, and tangent line at $s$. When $\kappa(s)=0$ the unit normal $n(s)$ is not defined and we set $\rho(s)=\infty$ and $c(s)=\infty=$ the point at infinity.

We say that an unordered pair $\{s, t\} \subset C$ is a tangent pair provided $X(s) \neq X(t)$ and $T_{s}=T_{t}$. A tangent pair $\{s, t\}$ is regular if $\kappa(s) \neq 0$ and $\kappa(t) \neq 0$. The sets of regular tangent pairs is divided into two subsets. Given a regular tangent pair $\{s, t\}$ we let $\{s, t\} \in \mathrm{I}_{t}$ iff $n(s)=-n(t)$, and $\{s, t\} \in \mathrm{II}_{t}$ iff $n(s)=n(t)$.

Given two vectors $v, w \in R^{2}$ let $[v, w]$ stand for the determinant of the $2 \times 2$ matrix with column vectors $v$ and $w$ in that order.

A crossing is an unordered pair $\{s, t\} \subset C$ such that $s \neq t$ and $X(s)$ $=X(t)$. A crossing $\{s, t\}$ is regular if $T_{s} \neq T_{t}$. Let $\mathcal{e}$ be the set of crossings.

A point $s \in C$ is an inflection point provided $\kappa(s)=0$. An inflection point $s$ is regular if $\kappa^{\prime}(s) \neq 0$. Let $F$ be the set of inflection points.

For any set $A$ denote the cardinality of $A$ by ${ }^{\sharp} A$.

REsult.

THEOREM 1. If all the tangent pairs, crossings and inflection points of $X$ are regular, then ${ }^{*} \mathrm{I}_{t},{ }^{*} \mathrm{II}_{t}$, ${ }^{*} \mathrm{C}$ and ${ }^{*} F$ are finite and

$$
\# \mathrm{II}_{t}=\# \mathrm{I}_{t}+\# \mathrm{C}+\frac{1}{2} \# F \text {. }
$$

Considering only $X$ which satisfy the hypothesis of Theorem 1, it is clear that ${ }^{*} \mathrm{II}_{t},{ }^{\sharp} \mathrm{I}_{t},{ }^{*} \mathrm{C}$ and $\frac{1}{2}{ }^{\sharp} F$ must be nonnegative integers and * $\mathrm{II}_{t}={ }^{*} \mathrm{I}_{t}+* \mathrm{e}+\frac{1}{2}{ }^{*} \mathrm{~F}$. Are these the only restrictions on the possible values for $\left({ }^{*} \mathrm{II}_{t},{ }^{*} \mathrm{I}_{t},{ }^{*} \mathrm{C}, \frac{1}{2} F\right)$ and, if not, what is the totality of all possible values for ${ }^{*} \mathrm{II}_{t},{ }^{*} \mathrm{I}_{t}$, $\left.{ }^{*} \mathrm{C}, \frac{1}{2}{ }^{*} F\right)$ with $X$ satisfying the hypothesis of Theorem 1? The answer to the first part of the question is no, but a complete answer to the second part is not known. However, we do have the following information. General examples show that all triplets $(a, b, c)$ with $a, b$ and $c-1$ nonnegative integers are possible values for $\left.{ }^{\sharp} \mathrm{I}_{t},{ }^{*} \mathrm{C}, \frac{1}{2}^{\sharp} F\right)$. The case with no inflection points is more interesting. It can be shown that if $\# F=0$ then ${ }^{*} I_{t}$ is even and ${ }^{*} I_{t}$ $\leqq 4\left({ }^{\sharp} \mathfrak{C}\right)^{2}+2\left({ }^{\sharp} \mathfrak{C}\right)$. A general example shows that all triplets of the form $(a, b, 0)$ with $a$ and $b$ nonnegative integers satisfying $a \leqq b^{2}-b$, and $a$ even, are possible values for $\left({ }^{\sharp} \mathrm{I}_{t},{ }^{\sharp} \mathrm{C}, \frac{1}{2}^{\sharp} F\right)$. This and other examples support the conjecture that if ${ }^{*} F=0$ then $I_{t} \leqq\left({ }^{*}\right)^{2}-{ }^{*}$. 
If this conjecture were established then in light of the above facts, the set of all possible $\left({ }^{*} \mathrm{II}_{t},{ }^{*} \mathrm{I}_{t},{ }^{\sharp} \mathfrak{C}, \frac{1}{2}{ }^{\sharp} F\right)$ would be completely determined.

OUtLINe OF PROOF. Parameterize the curve $X$ by arclength and thus represent it as a four times continuously differentiable function $X: R \rightarrow R^{2}$ of period $L=$ the total arclength of $X$. Consider the vector field $w(s, t)=(u(s, t), v(s, t))$ where

$$
u(s, t)=[\dot{X}(s), X(s)-X(t)]
$$

and

$$
v(s, t)=[X(t), X(t)-X(s)]
$$

$(\cdot=$ differentiation with respect to arclength). The critical points (zeros) of $w$ in the set $Q=\{(s, t) \mid 0 \leqq t \leqq 2 L$ and $t<s<t+L\}$ clearly correspond to tangent pairs and crossings. Furthermore, the index of a critical point $(s, t) \in Q$ is +1 if $\{s, t\} \in \mathrm{I}_{t} \cup C$, and is -1 if $\{s, t\}$ $\in \mathrm{II}_{t}$. The regularity conditions lead to the conclusion that the number of critical points in $Q$ is finite. Hence, we can find a $t_{0}, 0 \leqq t_{0} \leqq L$, and $\epsilon>0$ such that $A-B$ contains no critical points where $A$ $=\left\{(s, t) \mid t_{0} \leqq t \leqq t_{0}+L\right.$ and $\left.t<s<t+L\right\}$ and $B=\left\{(s, t) \mid t_{0}<t<t_{0}+L\right.$ and $t+\epsilon<s<t+L-\epsilon\}$. The winding number of $w$ along the boundary of $B$ transversed counterclockwise is found to be $-\#$. Theorem 1 then follows by setting the sum of the indices of the critical points in $B$ equal to this winding number.

FurTHER DEFINITIONS. We say that an unordered pair $\{s, t\} \subset C$ is a normal pair provided $X(s) \neq X(t)$ and $N_{s}=N_{t}$. An ordered pair $(s, t) \in C \times C$ is a $t n$-pair if $X(s) \neq X(t)$ and $T_{s}=N_{t}$. A normal pair $\{s, t\}$ is regular if $c(s) \neq c(t)$. A tn-pair $(s, t)$ is regular if $\kappa(s) \neq 0$ and $c(t) \neq X(s)$. The sets of regular normal pairs and regular $t n$-pairs are each divided into two subsets. The distinction between the two classes of regular normal pairs depends upon the positions of the two centers of curvature relative to each other and to the two points on the curve. Let $\{s, t\}$ be a regular normal pair and set $l=X(t)-X(s)$ and $\|l\|$ $=$ the norm of $l$. Let $\{s, t\} \in \mathrm{I}_{n}$ iff either

(1) $n(s)=-n(t)=l /\|l\|$ and $\rho(s)+\rho(t)<\|l\|$ or

(2) $n(s)=n(t)=l /\|l\|$ and $\rho(s)>\rho(t)+\|l\|$ or

(3) $n(s)=n(t)=-l /\|l\|$ and $\rho(t)>\rho(s)+\|l\|$ or

(4) $n(s)=-n(t)=-l /\|l\|$ or

(5) $\kappa(s)=0$ and $n(t)=l /\|l\|$ or

(6) $\kappa(t)=0$ and $n(s)=-l /\|l\|$.

Let $\mathrm{II}_{n}$ be the complementary subset to $\mathrm{I}_{n}$ of regular normal pairs.

Let $(s, t)$ be a regular $t n$-pair. Set $\sigma=1$ if 
$n(t)=(X(s)-X(t)) /\|X(s)-X(t)\|$ and $\rho(t)<\|X(s)-X(t)\| ;$

set $\sigma=-1$ in all other cases. Now, let $(s, t) \in \mathrm{I}_{t n}$ iff $\sigma[n(s)$, $(X(s)-X(t))]>0$, and $(s, t) \in \mathrm{II}_{t n}$ iff $\sigma[n(s),(X(s)-X(t))]<0$.

A crossing $\{s, t\}$ is biregular if $T_{s} \neq T_{t}$ and $N_{s} \neq T_{t}$. Let the scalar product of two vectors $v, w \in R^{2}$ be denoted by $(v, w)$. We assign an index to each biregular crossing $(s, t)$ as follows. If

$$
[\dot{X}(s), X(t)](\dot{X}(s), X(t))>0
$$

then set index $(s, t)=1$, and if $[\dot{X}(s), \dot{X}(t)](\dot{X}(s), \dot{X}(t))<0$ then set index $(s, t)=-1$.

We will want to consider another plane curve also given by a four times continuously differentiable function $Y: C^{\prime} \rightarrow R^{2}, Y^{\prime}(s) \neq 0$ all $s \in C^{\prime}$, where $C^{\prime}$ is another copy of the circle. The concepts of tangent pair, normal pair, $t n$-pair, and crossing and their regularity and distinguished subclasses carry over easily to ordered pairs $(s, t) \in C \times C^{\prime}$. We obtain in this way classes $\mathrm{I}_{t}^{\prime}, \mathrm{II}_{\ell}^{\prime}, \mathrm{I}_{n}^{\prime}, \mathrm{II}_{n}^{\prime}, \mathrm{I}_{t n}^{\prime}, \mathrm{II}_{t n}^{\prime}$, and $\mathfrak{e}^{\prime}$.

Similarly we may replace $C$ by any compact $C^{4}$, one-dimensional closed manifold and consider $X$ to be a $C^{4}$ immersion. When we interpret $C$ in this new way the sets defined above will be called $I_{t}^{\prime \prime}$, $\mathrm{II}_{t}^{\prime \prime}, \mathrm{I}_{n}^{\prime \prime}$, etc.

FURTHER RESULTS.

THEOREM 2. If all the normal pairs and crossings of $X$ are regular, then ${ }^{*} \mathrm{I}_{n},{ }^{*} \mathrm{II}_{n}$ and $\# \mathfrak{C}$ are finite and

$$
\mathrm{FI}_{n}=\sharp \mathrm{I}_{n}+\sharp \mathrm{C} .
$$

THEOREM 3. If all the tn-pairs of $X$ are regular and all the crossings biregular then $\mathrm{I}_{t n}, \mathrm{II}_{t n}$ and $\mathbb{E}$ are finite and

$$
\sharp \mathrm{II}_{t n}=\sharp \mathrm{I}_{t n} \text {. }
$$

THEOREM 4. If all the tangent pairs and crossings between $X$ and $Y$ are regular, then ${ }^{\#} \mathrm{I}_{t}^{\prime},{ }^{*} \mathrm{II}_{t}^{\prime}$, and ${ }^{*} \mathfrak{C}^{\prime}$ are finite and

$$
\mathrm{II}_{t}^{\prime}=\sharp \mathrm{I}_{t}^{\prime}+\sharp \mathcal{C}^{\prime} \text {. }
$$

TheOREM 5. If all the normal pairs and crossings between $X$ and $Y$ are regular, then ${ }^{\#} \mathrm{I}_{n}^{\prime},{ }^{\prime \prime} \mathrm{II}_{n}^{\prime}$, and ${ }^{\#} \mathrm{C}^{\prime}$ are finite and

$$
* \mathrm{II}_{n}^{\prime}=\sharp \mathrm{I}_{n}^{\prime}+\# \mathbb{C}^{\prime} \text {. }
$$

THEOREM 6. If all the tn-pairs between $X$ and $Y$ are regular and all the crossings between $X$ and $Y$ are biregular, then ${ }^{\prime} \mathrm{I}_{t n}^{\prime}, " \mathrm{I}_{t n}^{\prime}$ and ${ }^{*} \mathcal{C}^{\prime}$ are finite and 


$$
\sharp \mathrm{II}_{t n}^{\prime}=\mathrm{I}_{t n}^{\prime}+\sum_{p \in \mathbb{e}^{\prime}} \text { index } p .
$$

THEOREM 7. Theorems 1, 2, and 3 hold with $X$ interpreted as an immersion of a compact, $C^{4}$, not necessarily connected, closed onedimensional manifold. That is, under appropriate regularity conditions

$$
\begin{aligned}
& \# \mathrm{II}_{t}^{\prime \prime}=\sharp \mathrm{I}_{t}^{\prime}+\# \mathrm{C}^{\prime \prime}+\frac{1}{2} \sharp F^{\prime \prime}, \\
& \sharp \mathrm{II}_{n}^{\prime \prime}=\sharp \mathrm{I}_{n}^{\prime \prime}+\# \mathrm{C}^{\prime \prime}, \\
& \sharp \mathrm{II}_{t n}^{\prime \prime}=\sharp \mathrm{I}_{t n}^{\prime \prime},
\end{aligned}
$$

and the above quantities are finite.

Proofs of Theorems 2-7. The proofs of Theorems 2 through 6 are similar to the proof of Theorem 1 and make use of the vector fields $w_{1}=\left(u_{1}, v_{1}\right)$ in the $t n$-pairs cases, and $w_{2}=\left(u_{2}, v_{2}\right)$ in the normal pairs cases, where

$$
\begin{aligned}
u_{1}(s, t) & =[\dot{X}(s), X(s)-X(t)], \\
v_{1}(s, t) & =(\dot{X}(t), X(t)-X(s)), \\
u_{2}(s, t) & =(\dot{X}(s), X(s)-X(t)), \\
v_{2}(s, t) & =(\dot{X}(t), X(t)-X(s)) .
\end{aligned}
$$

Theorem 7 is just a corollary to Theorems 1 through 6 .

REMARK. Note that $w_{2}(s, t)=\frac{1}{2} \operatorname{grad}\|X(s)-X(t)\|^{2}$. This leads to an alternate proof of Theorems 2 and 5 using Morse theory. Extreme points for $\|X(s)-X(t)\|^{2}$ correspond to points in $\mathrm{I}_{n}$ and from this it follows that $\mathrm{I}_{n} \geqq 1$.

Extensions. The regularity and smoothness hypotheses above can be considerably weakened, and cusps and lines going off to infinity can be included in the theory. Similar results hold for curves on the sphere $S^{2}$ and in $R^{3}$. Also, the above techniques yield formulas involving special $n$-tuplets of points on $X$.

University of California, Berkeley, California 94720 